\title{
AETIOLOGY AND EPIDEMIOLOGICAL PROFILE OF CORNEAL ULCERATION IN TERTIARY CARE CENTRE
}

\author{
Hemalatha Krishnamurthy1, Manjula Devi N², Satish ${ }^{3}$, Shyam ${ }^{4}$, Shreedevi Badagi5 ${ }^{5}$, Sujatha ${ }^{6}$, Mohammed Abdul Khayoom ${ }^{7}$, \\ Chandresh Suthar ${ }^{8}$
}

${ }^{1}$ Associate Professor, Department of Ophthalmology, Mysore Medical College and Research Institute, Mysore. ${ }^{2}$ Associate Professor, Department of Ophthalmology, Mysore Medical College and Research Institute, Mysore. 3 Professor and HOD, Department of Ophthalmology, Mysore Medical College and Research Institute, Mysore. 4 Junior Resident, Department of Ophthalmology, Mysore Medical College and Research Institute, Mysore. $5 J u n i o r$ Resident, Department of Ophthalmology, Mysore Medical College and Research Institute, Mysore. 6 Junior Resident, Department of Ophthalmology, Mysore Medical College and Research Institute, Mysore. 7 Junior Resident, Department of Ophthalmology, Mysore Medical College and Research Institute, Mysore. 8Junior Resident, Department of Ophthalmology, Mysore Medical College and Research Institute, Mysore.

\section{ABSTRACT}

\section{BACKGROUND}

The epidemiology of corneal blindness is much complicated and encompasses a wide variety of inflammatory and infectious eye diseases that cause corneal scarring that ultimately leads to functional blindness. In addition, the prevalence of corneal disease varies from one population to another and from country to country.

The purpose of this study was to study the epidemiology of corneal ulcer in a tertiary care centre and identify the various risk factors, predisposing factors in corneal ulcers and the microbial spectrum causing the ulcers.

\section{MATERIALS AND METHODS}

This descriptive study was conducted in a tertiary care hospital in patients attending Department of Ophthalmology, K. R. Hospital, Mysore Medical College and Research Institute, Mysore, in a period of 1 year from April 2016 to March 2017. Corneal epithelial loss with suppuration associated with signs of inflammation with or without hypopyon was taken as corneal ulcer. All patients diagnosed with corneal ulcer were included. Complete history, detailed ocular examination with risk factors were recorded. Corneal scrapings collected from the edge of the ulcer were processed for microbiological analysis at K. R. Hospital.

\section{RESULTS}

Total number of patients included in the study was 123 , out of which there were $73(59.35 \%)$ males and $50(40.65 \%)$ females with male preponderance. The rural population (88.62\%) was affected much more commonly than the urban $(11.38 \%)$. Trauma was the most common predisposing factor leading to corneal ulcers (94.30\%). Sugarcane/paddy stick injury (34.91\%) being the most common mode of trauma. Streptococcus pneumoniae was the most commonly isolated Gram-positive bacterial organism representing $22(41.51 \%)$ and Pseudomonas spp. was the most frequently occurring Gram-negative organism and it was isolated from 11 cultures (20.75\%). Among 35 cases with pure fungal growths, fusarium was found commonest 21 (17.07\%).

\section{CONCLUSION}

Infective keratitis being the leading cause for monocular blindness, early clinical suspicion, diagnostic laboratory procedures and appropriate timely therapy has tremendous role in reducing ocular morbidity.

\section{KEYWORDS}

Bacterial; Corneal Abrasion; Fungal; Keratitis; Trauma.

HOW TO CITE THIS ARTICLE: Krishnamurthy H, Devi MN, Satish K, et al. Aetiology and epidemiological profile of corneal ulceration in tertiary care centre. J. Evolution Med. Dent. Sci. 2017;6(76):5463-5468, DOI: 10.14260/Jemds/2017/1185

\section{BACKGROUND}

Worldwide diseases affecting the cornea are a major cause of blindness, second only to cataract in overall importance.[1] The epidemiology of corneal blindness is much complicated and encompasses a wide variety of inflammatory and infectious eye diseases that cause corneal scarring that ultimately leads to functional blindness. In addition, the prevalence of corneal disease varies from one population to another and from country to country. ${ }^{[2]}$ Corneal ulcer is a

Financial or Other, Competing Interest: None.

Submission 09-08-2017, Peer Review 09-09-2017,

Acceptance 15-09-2017, Published 21-09-2017.

Corresponding Author:

Manjula Devi N,

Associate Professor, Department of Ophthalmology,

Mysore Medical College and Research Institute, Mysore.

E-mail:dr.hemalatha_k@yahoo.in

DOI: $10.14260 /$ jemds/2017/1185

\section{(c) $($ ) $\$$}

sight threatening disorder in all age groups and both in males and females worldwide. It may resolve without any squeal, progress to perforation with its resultant consequences or leave behind a corneal opacity, which may lead to loss of vision if it is present centrally. ${ }^{[3]}$

Ocular trauma and corneal ulceration are significant causes of corneal blindness that are often under-reported, but may be responsible for 1.5 - 2.0 million new cases of monocular blindness every year, posing a major public health problem. As National Public Health Programmes have become more effective in reducing the prevalence of traditional causes of corneal blindness such as trachoma, leprosy and onchocerciasis, so ocular trauma and corneal ulceration have become relatively more important. In developing countries, infectious keratitis is the leading cause of corneal blindness.[1] Central corneal ulceration is an important cause of uniocular blindness in developing 
countries. These findings have been confirmed by surveys in Africa and Asia.[2]

The various risk factors for corneal ulcers and its mode of presentations are rife in literature, but they vary from region to region as it depends on the socioeconomic status, culture, occupation and habits of a particular region.[3] To prevent corneal ulcers, the initial step is to determine its epidemiology in different parts of the country and in different states by the use of which risk factors can be identified and measures to prevent them implemented.[1]

Corneal damage by the pathogens may be directly or by release of toxins and enzymes or by activating the host immune system. For the majority of microorganisms, an intact corneal epithelium acts as a barrier. A breach in the corneal epithelium either due to penetrating or perforating ocular trauma allows penetration of the microorganisms. ${ }^{[4]}$ In India, Corneal blindness is a major problem, which adds a substantial burden to the community in general and health care resources. Further persons with corneal blindness are usually of younger age group compared with cataract persons, resulting in more impact from corneal blindness. In India approximately 6.8 million people have uniocular corneal blindness with vision less than $20 / 200$ in at least one eye and of these about a million have bilateral corneal blindness. It is expected that the no. of corneal blind people in India will increase to a staggering 10.6 million by 2020.[5] Due to these reasons, this study was taken up to evaluate the various risk factors, age and sex predilections and the type of microbes causing the ulcers, so that a systematic approach may be adopted in preventing and treating this sight threatening disorder in these southern regions of India.

\section{Objectives}

- Study the epidemiology of corneal ulcer in a tertiary care centre.

- The various risk factors and predisposing factors in corneal ulcers.

- The microbial spectrum causing the ulcers.

\section{MATERIALS AND METHODS}

This descriptive study was conducted in a tertiary care hospital in patients attending outpatient/inpatient in the Department of Ophthalmology, K. R. Hospital, MMC and RI, Mysore. Total 123 cases were diagnosed to have corneal ulcer in a period of 1 year from April 2016 to March 2017 were enrolled. Corneal epithelial loss with suppuration associated with signs of inflammation with or without hypopyon was taken as corneal ulcer.

\section{Inclusion Criteria}

All patients were diagnosed with corneal ulcer in this centre.

\section{Exclusion Criteria}

Typical viral ulcers, Mooren's ulcers, Marginal ulcers, Interstitial keratitis, Sterile neurotrophic ulcers and any ulcers associated with autoimmune conditions.

Patient's data like age and sex were noted. In all possible cases, visual acuity was recorded and in all cases detailed slit lamp examination of the anterior segment was done noting the size of ulcer, depth and extent of ulcer with infiltrations, presence/absence of hypopyon, scleral extension and corneal thinning. Local eye conditions predisposing to corneal ulceration like lagophthalmos, dacryocystitis, trichiasis, surgery and corneal opacity/degeneration were noted. For all these patients before treatment scrapings were taken from the ulcer margin and base, viewing through slit lamp using a 27-gauge needle after anaesthetising with topical 4\% xylocaine eye drops. These were taken on 2 glass slides, one for Gram's stain and the other for immediate $10 \% \mathrm{KOH}$ mount. The scrapings were inoculated on to the blood agar, chocolate agar, MacConkey agar and Sabouraud's dextrose agar (SDA) in multiple streaks. They were incubated overnight. Culture of bacteria was confirmed by growth on blood agar, chocolate agar and MacConkey agar followed by standard biochemical tests. SDA was inspected for up to ten days and then was declared as fungal culture negative or positive. Growth of fungus was grossly identified by colonial morphology, pigment production and microscopically by lactophenol cotton blue staining. Fungal ulcer was diagnosed when the following criteria (any one) were met: Correlation between $\mathrm{KOH}$ mount and SDA culture, growth seen on $>1 \mathrm{C}$ streaks and similar growth on $>1$ medium. All investigations like microbiological analysis was done at Department of Microbiology, K. R. Hospital, Mysore. All investigation reports were collected from microbiology department for both in/outpatients. Inpatient's case record maintained at ophthalmology department was taken; and outpatient's complete history, clinical findings investigations and management was noted and recorded.

\section{RESULTS}

The criteria for clinical diagnosis was corneal epithelial defect with underlying stromal infiltration and suppuration with signs of inflammation in all 123 cases. In addition, at the initial examination 36 patients $(29.26 \%)$ had a documented hypopyon.

Total number of patients included in the study was 123 out of which there were 73 (59.35\%) males and 50 (40.65\%) females. The most commonly affected age group was $46-60$ years.

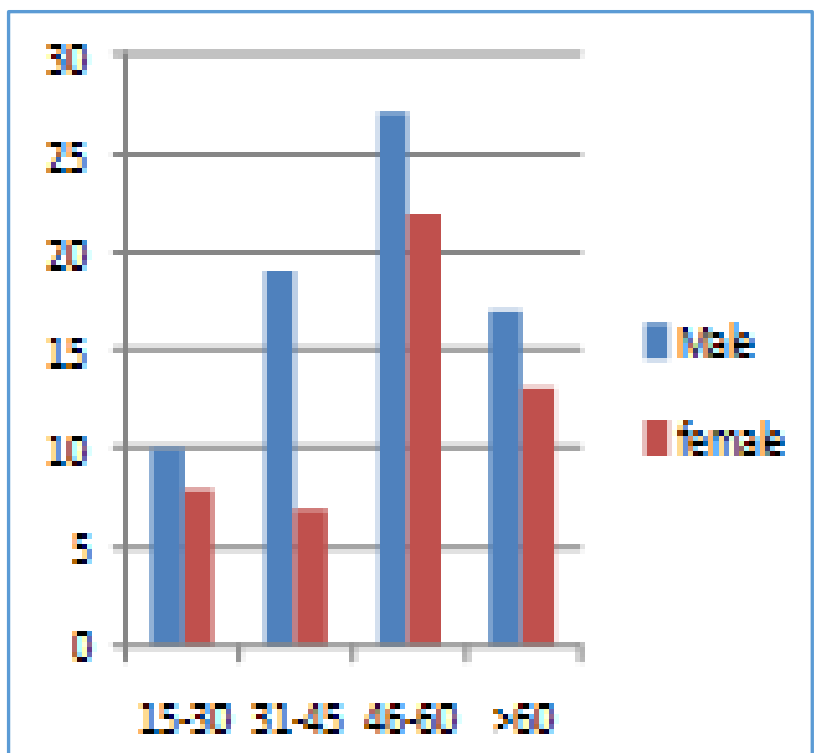

Graph 1. Showing Age and Sex Wise Distribution of Corneal Ulcer 
Overall, the ratio of male-to-female patients with corneal ulceration was 1.46:1. The rural population (88.62\%) was affected much more commonly than the urban (11.38\%). Trauma was the most common predisposing factor leading to corneal ulcers $(94.30 \%)$ followed by dacryocystitis $(3.30 \%)$ and then use of steroids $(0.80 \%)$.

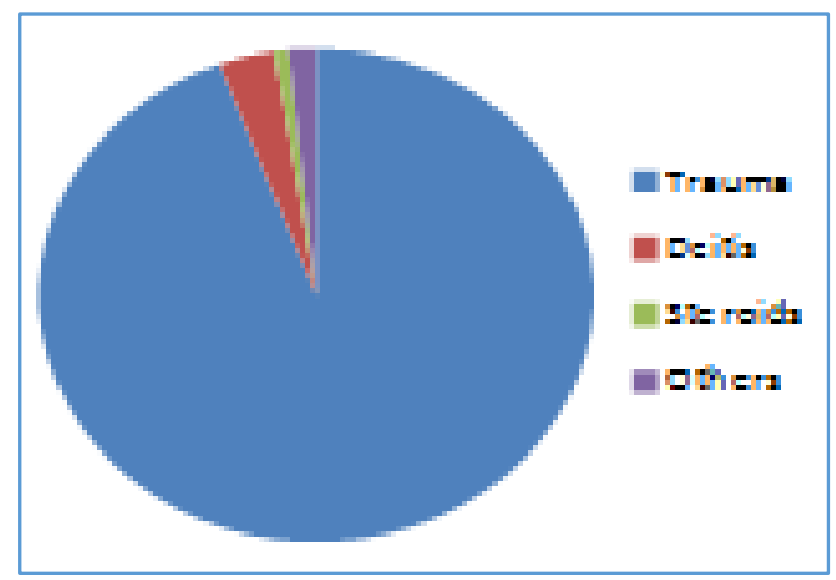

Graph 2. Showing Predisposing Factors for Corneal Ulcer

Sugarcane/paddy stick (34.91\%) was the most common mode of trauma followed by tree branch/thorn injury (27.64\%), then foreign body (17.89\%) and stone injuries $(16.26 \%)$ and animal tail injuries $(3.30 \%)$ not being left far behind.

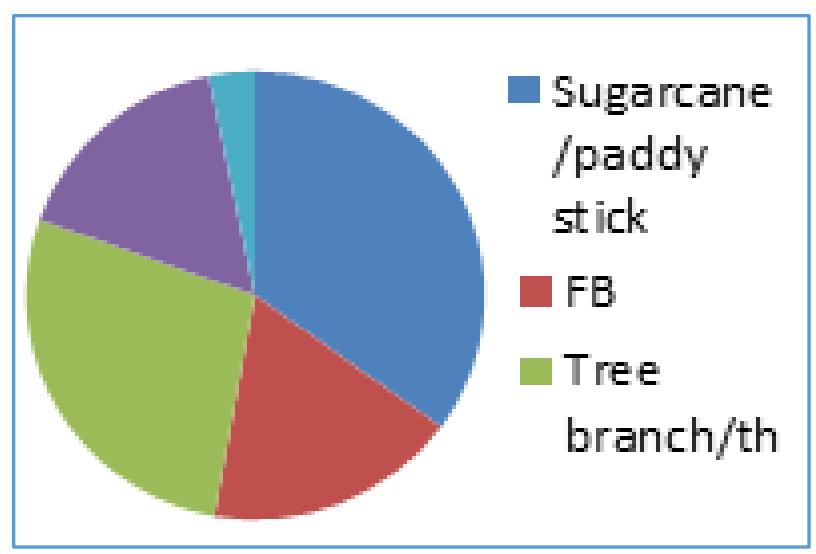

\section{Graph 3. Showing Mode of Trauma Predisposing for Corneal Ulcer}

Microbiological analysis was positive in 88 cases (71.45\%), showed a pure bacterial infections in 44 (35.77\%) patients and pure fungal infection in 35 (28.46\%) patients. Mixed bacterial and fungal infection was found in 9 (7.32\%) patients, whereas in 35 (28.64\%) patients the culture remained negative (Graph 4).

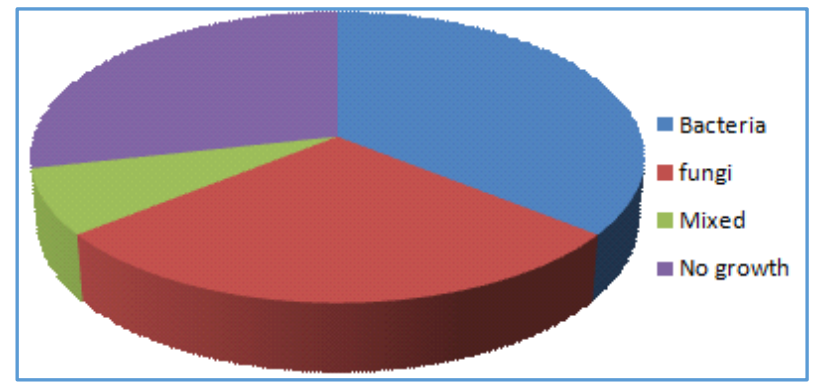

Graph 4. Showing Microbiological Analysis
Among the pure bacterial ulcers, there were 34 (27.64\%) gram positive cocci and $10(8.13 \%)$ gram negative cocci. Among 35 cases with pure fungal growths, fusarium was commonest $21(17.07 \%)$ followed by Aspergillus 9 (4.07\%) and candida 5 (7.32\%) Table 1.

\begin{tabular}{|c|c|c|c|c|}
\hline Organism & Species & $\begin{array}{c}\text { No. of } \\
\text { Cases }\end{array}$ & \% & \\
\hline Bacteria & GPC & 34 & $27.64 \%$ & $35.77 \%$ \\
\hline & GNC & 10 & $8.13 \%$ & \\
\hline Fungi & Fusarium & 21 & $17.07 \%$ & $28.46 \%$ \\
\hline & Candida & 9 & $7.32 \%$ & \\
\hline & Aspergillus & 5 & $4.07 \%$ & \\
\hline Mixed & \multicolumn{5}{c|}{} & 35 & $28.46 \%$ & $28.46 \%$ \\
\hline No growth & Table 1. Showing various Microbiological Profile in 123 \\
Cases of Corneal Ulcers GPC (Gram-Positive Cocci), GNC \\
(Gram-Negative Cocci) \\
\hline
\end{tabular}

A total of 53 bacterial organisms were cultured from 123 corneal ulcers (Table 5). Of the 53 isolates, pure bacterial were 34 (27.64\%) Gram positive and 10 (8.13\%) Gram negative. Streptococcus pneumoniae was the most commonly isolated gram-positive bacterial organism representing 22 (41.51\%) and Pseudomonas spp. The most frequently occurring Gram-negative organism was isolated from 11 cultures $(20.75 \%)$ followed by the next most commonly isolated Gram-positive organism Corynebacterium xerosis with 7 (13.21\%) positive cultures. Staphylococcus aureus from 4 patients (5.4\%), Staphylococcus epidermidis from 3 patients was cultured (10.2\%) and Bacillus spp., Nocardia and Streptococcus viridans were cultured in 1 case each. Other Gram-negative organisms infrequently isolated included Moraxella spp., Haemophilus influenza and Proteus mirabilis. Of these 53 cultures, 9 were mixed with fungi.

\begin{tabular}{|c|c|}
\hline Gram Positive Organisms & \\
\hline Corynebacterium xerosis & 7 \\
\hline Streptococcus pneumonia & 22 \\
\hline Staphylococcus aureus & 4 \\
\hline Streptococcus viridans & 1 \\
\hline Bacillus spp. & 1 \\
\hline Nocardia asteroides & 1 \\
\hline Staphylococcus epidermidis & 3 \\
\hline Gram Negative Organisms & 11 \\
\hline Pseudomonas spp. & 1 \\
\hline Moraxella spp. 3 & 1 \\
\hline Haemophilus influenzae & 1 \\
\hline Proteus mirabilis &
\end{tabular}

Table 2. Showing various Species of Bacteria in 53 Cases

\begin{tabular}{|c|c|c|}
\hline Fungi & Fusarium & 21 \\
\hline & Candida & 9 \\
\hline \multicolumn{2}{|c|}{ Aspergillus } & 5 \\
\hline \multicolumn{2}{|c|}{ Table 3. Showing various Fungi in 35 Cases } \\
\hline
\end{tabular}

Of all the fungal organisms cultured from patients with ulcer, 35 cultures $(28.46 \%)$ were pure isolates and 9 (7.32\%) were mixed with a corresponding single species of bacteria. 


\begin{tabular}{|c|c|c|l|c|}
\hline Mixed & Bacteria & $\begin{array}{c}\text { No. of } \\
\text { Cases }\end{array}$ & Fungi & $\begin{array}{c}\text { No. of } \\
\text { Cases }\end{array}$ \\
\hline & $\begin{array}{c}\text { Streptococcus } \\
\text { Pneumoniae }\end{array}$ & 2 & Fusarium & 5 \\
\hline & $\begin{array}{c}\text { Corynebacterium } \\
\text { xerosis }\end{array}$ & 4 & Aspergillus & 3 \\
\hline & $\begin{array}{c}\text { Staphylococcus } \\
\text { aureus }\end{array}$ & 3 & Candida & 1 \\
\hline Table 4. Showing Mixed Bacterial and Fungal Growth in 9 \\
Cases
\end{tabular}

Surgical intervention like therapeutic keratoplasty continues to be an important mode of management. In cases with central corneal ulcer perforation, penetrating keratoplasty was performed in 28 cases with advanced disease at presentation, wherein no response to energetic medical therapy was observed or when a large perforation is present; 12 cases underwent dacryocystectomy.

\section{DISCUSSION}

Corneal scarring has been the second most common cause of blindness and visual disability in many developing nations in Asia, Africa and the Middle East.[2] Thylefors ${ }^{[6]}$ stresses the importance of superficial corneal trauma in agricultural work, that frequently leads to rapidly progressing corneal ulceration and visual loss which has been overlooked as a worldwide cause of monocular blindness. Whitcher JP et al reports that up to $5 \%$ of all blinding conditions are directly related to ocular trauma and subsequent infection. ${ }^{[7]}$

Suppurative corneal ulcer with complications resulting with sequelae is a vision threatening disorder in all age groups and both in males and females worldwide.[3] A detailed clinical history coupled with clinical examination would identify the predisposing factors for microbial keratitis and be beneficial for early and energetic management. Early management of all infective keratitis and its complications is essential to reduce the ocular morbidity resulting from corneal blindness.

The prevalence of corneal blindness varies from one population to another and country to country depending on many factors, such as availability and general standards of eye care. ${ }^{[8]}$ The prevalence of visual disability from corneal opacity varies from one geographical location to another. In south India, the incidence of corneal ulceration is more than 10 times higher (11.3 per 10000$)$ than in a comparable population in the USA and in Madurai district alone reports an estimated 3500 individuals being affected each year. ${ }^{[2,9]}$ In children and young adults, Corneal disease resulting in corneal scarring is also a common cause of monocular and bilateral blindness. Visual loss is 20 times higher than in industrialised countries in high-risk groups in some parts of Asia and Africa.[1] Corneal ulceration in developing countries has only recently been recognised as a "silent epidemic" [7,1,2] Both trauma and ulceration are usually monocular and affected persons are therefore not characterised as totally blind, but only as visually disabled.[1]

Total number of the patients included in the study was 123 , out of which there were $73(59.35 \%)$ males and 50 $(40.65 \%)$ females showing a male preponderance. Srinivasan et al have reported that males in their sixth decade of life are particularly at risk. ${ }^{[2]}$ The most commonly affected age group was between $46-60$ years, which is in concordance with another study by Dr. Chhangte L et al.[10] The age group of 41 - 60 years was more affected consistent with the results of Cameron et al[11] in Sydney and Das et al[12] in Kolkata. In males, the predominance of corneal ulceration was most pronounced in the middle years with an overall ratio of maleto-female patients of 1.46 to 1 . This concords with the Madurai study where the overall ratio of male-to-female patients with corneal ulceration reported was 1.6: 1, the same ratio reported by Gonzales et al.[2,9]

Both sexes are prone to develop corneal ulcers in the middle decades of life when presumably they are more physically active and are at higher risk for corneal injury. The same findings are observed in our study also.[2] The rural population $(88.62 \%)$ was affected much more commonly than the urban (11.38\%).

The majority of corneal ulcer patients were farmers that is agricultural workers 72 and farm labourers 24 (78.05\%), an occupation profile similar to Srinivasan et al from Madurai $(78.8 \%)^{[2]}$ and Upadhyaya et al from Nepal $(72 \%),[13]$ but in marked contrast with Ghana where only $16.1 \%$ of the patients were involved in agricultural activity.[14] Madurai study by Srinivasan et al reflects agricultural workers being more at risk and had corneal ulcers.[2] The study by Chhangte L et al on 76 patients, keratitis showed that ocular trauma was the most common predisposing factor in $55(72.4 \%)$ patients $(\mathrm{p}=0.001)$, followed by co-existing ocular disorder in 7 (9.2\%) patients.[10] The same was reflected in our study having trauma as a predisposing factor in 116 cases (94.30\%), while the other factors accounted only for 7 $(5.60 \%)$ cases. This can be attributed to an increased outdoor activity of males, especially in the working age group. To contrast this finding, a study done in China showed more women getting affected than men, most of them being over 60 years of age.[15] This could be due to the fact that women in China are more active in the agricultural sector. Most of our patients were engaged in heavy labour, either in an agricultural setting or in construction.

In our study, out of 123 patients 88 patients (71.54\%) showed a positive culture. Srinivasan et al reports positive culture in $297(68.4 \%)$ of the 434 corneas that were inoculated and this nearly concords with our study.[2] This concords favourably with a recent study in Ghana[14] where $57.3 \%$ of all cultures were positive, but higher positive culture of $80 \%$ isolation rate reported from Nepal by Upadhyaya et al[13] and rate of $81.7 \%$ reported by Dunlop et al[16] in Bangladesh.

Out of the positive culture $44(35.77 \%)$ were pure bacterial, 35 (25.46\%) were pure fungal and 9 (7.32\%) mixed. Bacterial positive cultures being more in number compared to fungal. The same was mirrored in a similar study in South India by Srinivasan et al, ${ }^{[2]}$ who isolated equal numbers of bacterial (47.1\%) and fungal (46.8\%) agents causing infectious keratitis with $5.1 \%$ cases having mixed infections. Same study reported $47.1 \%$ Fusarium spp. and $16.1 \%$ Aspergillus spp. out of $51.9 \%$ fungal isolated cases.[2]

Fungi alone or in combination with bacteria were isolated from $35.78 \%$ of all culture positive patients from our series. Fusarium spp. accounted for $59.09 \%$ of all fungal isolates, a little higher than Madurai studies. Our study concords with study by Ranjini et al,[4] who showed that among the fungal growth Fusarium was found to be common, whereas a study by Katara et al in Gujarat showed Aspergillus as the dominant 
isolate.[17] Liesegang and Forster from south Florida[18] and Hagan et al from Ghana[14] reports similar pattern of fungal organisms dominated by Fusarium spp. Reports from South Florida, $35 \%$ of the isolated organisms were fungi with predominance of Fusarium spp. accounting for $61 \% .{ }^{[18]}$ In Ghana, fungi alone or in combination with bacteria were isolated from $56 \%$ of all culture positive patients. Fusarium spp. accounted for $52 \%$ of all fungal isolates, which is similar to our study.[14] The climates of south India are similar in many respects to the climate of south Florida and Ghana, which may explain the corresponding pattern of fungal organisms. Studies in the South Indian region have shown a higher incidence of Fusarium as compared to studies in the northern or western India.[9] By contrast, in most of the world Aspergillus spp. or Candida spp. are the predominant fungal pathogens responsible for mycotic keratitis.[19] In Nepal with temperate climate, Upadhyaya et al[13] found that Aspergillus spp. accounted for $47 \%$ of all fungal pathogens followed by Candida spp. (13.2\%) and Fusarium spp. (11.7\%). In south India, it is very obvious that fungal keratitis is an enormous public health problem. There is difference in the isolation rates of these fungal pathogens, which may be explained by the differences in the climate and the natural environment of individual regions as Aspergillus spores tolerate dry and hot weather conditions better than the other fungi. Fusarium spp., one of the most virulent ocular pathogens responsible for majority of corneal ulcers needs more effective methods of management to reduce the load of preventable corneal blindness.[2]

In our study, microbiological analysis showed pure bacterial infections in $44(35.77 \%)$ patients and mixed bacterial and fungal infections in $9(7.32 \%)$ patients. Of the 53 isolates, pure bacterial were $34(27.64 \%)$ Gram positive and $10(8.13 \%)$ Gram negative. Streptococcus pneumoniae was the most commonly isolated bacterial organism representing $22(41.51 \%)$ of all positive bacterial cultures followed by Corynebacterium xerosis with 7 (13.21\%) positive cultures. The most common isolate streptococcus pneumoniae is attributed to the large numbers of chronic dacryocystitis that was detected in this study, where pneumococci were the prime isolate. Pseudomonas spp. was isolated from 11 cultures $(20.75 \%)$ and was the most frequently occurring Gram-negative organism. Our study concords with study by Srinivasan et al.[2] They reported Streptococcus pneumoniae as the most commonly isolated gram-positive bacterial organism representing (44.3\%) and Pseudomonas spp. (14.4\%) was the most frequently reported Gram-negative organism. Bacteria account for $65 \%-90 \%$ of corneal infections with Staphylococcus aureus, $\mathrm{S}$. pneumoniae and Pseudomonas aeruginosa accounting for more than $80 \%$ of bacterial keratitis as stated by Forbes.[20] Keshav et al[3] showed $58.3 \%$ isolates as pseudomonas, in another study by Leck[21] et al pseudomonas was found to be the predominant isolate amongst the bacterial ulcers in Ghana. Several other studies from Upadhyaya et al reported $31.1 \%$ pneumococci, the most common isolate.[13] The changes in bacterial flora within the geographical area are influenced by climate and environment.

With such a dismal prospect for both conservative medical and surgical treatment for corneal ulcers, the public health solution for this enormous problem is logically a strategy for prevention. The majority of corneal ulcers follow the occurrence of often trivial corneal abrasions. ${ }^{[2]}$ Patients who presents with corneal abrasions without signs of infection, these patients treated prophylactically with broadspectrum ophthalmic ointment three times a day for three days can prevent the occurrence of a corneal ulcer. This has already been reported by Upadhyaya et al[13] of 442 corneal abrasions that were treated in this manner, 96\% healed without developing an ulcer. Post-traumatic corneal ulceration can be prevented by timely application of broadspectrum ointment to eyes with corneal abrasions, but prophylaxis must be started within 18 hours after injury for maximum benefit to be obtained.

\section{CONCLUSION}

Infective keratitis being the leading cause for monocular blindness, early clinical suspicion, diagnostic laboratory procedures and appropriate timely therapy has tremendous role in reducing ocular morbidity. In the population at risk, undoubtedly the most common predisposing factor for corneal ulceration in south India is a history of corneal injury, which if untreated timely develops into corneal ulcer. Especially for the farmers in agriculture work at risk, public health programme to be planned in an efficient manner for the rapid diagnosis, treatment and ultimately the prevention of corneal ulceration. In the developing world, this approach has important public health implications for the treatment and prevention of corneal ulcers.[1]

\section{REFERENCES}

[1] John WP, Srinivasan M, Madan UP. Corneal blindness: a global perspective. Bull World Health Organ 2001;79(3):214-21.

[2] Srinivasan M, Gonzales CA, George C, et al. Epidemiology and aetiological diagnosis of corneal ulceration in Madurai, South India. British Journal of Ophthalmology 1997;81(11):965-71.

[3] Keshav BR, Zacheria G, Ideculla T, et al. Epidemiological characteristics of corneal ulcers in south Sharqiya region. Oman Med J 2008;23(1):34-9.

[4] Ranjini CY, Vandana VW. Microbial profile of corneal ulcers in a tertiary care hospital in South India. J Ophthalmic Vis Res 2016;11(4):363-7.

[5] Ravinder K, Madhav VM, Archana J, et al. Clinical evaluation of corneal ulcer among patients attending teaching hospital. International Journal of Contemporary Medical Research 2016;3(4):949-52.

[6] Thylefors B, Negrel AD, Segaram PR, et al. Available data on blindness (update 1994). Ophthalmic Epidemiology 1995;2(1):5-39.

[7] Whitcher JP. Corneal ulceration in the developing world-a silent epidemic. British Journal of Ophthalmology 1997;81(8):622-3.

[8] Smith GT, Taylor HR. Epidemiology of corneal blindness in developing countries. Refractive and Corneal Surgery 1991;7(6):436-9.

[9] Gonzales CA, Srinivasan M, Whitcher JP, et al. Incidence of corneal ulceration in Madurai district, 
South India. Ophthalmic Epidemiology 1996;3(3):15966.

[10] Chhangte L, Pandey S, Umesh. Epidemiological and microbiological profile of infectious corneal ulcers in tertiary care centre, kumaon region, Uttarakhand. International Journal of Scientific and Research Publications 2015;5(2):1-5.

[11] Cameron NL, Pham JN, Paul BR, et al. Bacteria commonly isolated from keratitis specimen retain antibiotic susceptibility to fluoroquinolones and gentamicin plus cephalothin. Clin Exp Ophthalmol 2006;34(1):44-50.

[12] Das S, Konar J. Bacteriological profile of corneal ulcer with references to antibiotic susceptibility in a tertiary care hospital in West Bengal. IOSR J Dent Med Sci 2013;11:72-5.

[13] Upadhyay MP, Karmacharya PC, Koirala S, et al. Epidemiologic characteristics, predisposing factors, and etiologic diagnosis of corneal ulceration in Nepal. Am J Ophthalmol 1991;111(1):92-9.

[14] Hagan M, Wright E, Newman M, et al. Causes of suppurative keratitis in Ghana. $\mathrm{Br} \mathrm{J}$ Ophthalmol 1995;79(11):1024-8.

[15] Cao J, Yang Y, Yang W, et al. Prevalence of infectious keratitis in central China. BMC Ophthalmol 2014;14:43.
[16] Dunlop AA, Wright ED, Howlader SA, et al. Suppurative corneal ulceration in Bangladesh. A study of 142 cases examining the microbiological diagnosis, clinical and epidemiological features of bacterial and fungal keratitis. Aust NZ J Ophthalmol 1994;22(2):105-10.

[17] Katara RS, Patel ND, Sinha M. A clinical microbiological study of corneal ulcer patients at western Gujarat, India. Acta Med Iran 2013;51(6):399-403.

[18] Liesegang TJ, Forster RK. Spectrum of microbial keratitis in South Florida. Am J Ophthalmol 1980;90(1):38-47.

[19] Jones BR. Principles in the management of oculomycosis. XXXI Edward Jackson memorial lecture. Am J Ophthalmol 1975;79(5):719-51.

[20] Infections of the eyes, ears and sinuses. In: Forbes BA, Sahm DF, Weissfeld AS, (eds). Baileyand scott's diagnostic microbiology. $12^{\text {th }}$ edn. St. Louis: Mosby 2007:832-7.

[21] Leck AK, Thomas PA, Hagan M, et al. Aetiology of suppurative corneal ulcers in Ghana and south India and epidemiology of fungal keratitis. Br J Ophthalmol 2002;86(11):1211-5. 